\title{
Two Silver Coordination Network Compounds with
}

\section{Colorful Photoluminescence}

Dandan Yang, ${ }^{a}$ Wenlong Xu, ${ }^{a}$ Xiaowei Cao, ${ }^{a}$ Shaojun Zheng, ${ }^{b}$ Jiangang He, ${ }^{b}$ Qiang Ju, ${ }^{a}$ Zhenlan Fang, $^{a}{ }^{*}$ and Wei Huang ${ }^{a}$.

${ }^{a}$ Key Laboratory of Flexible Electronics (KLOFE) \& Institute of Advanced Materials (IAM), Jiangsu National Synergetic Innovation Center for Advanced Materials (SICAM), Nanjing Tech University (NanjingTech), 30 South Puzhu Road, Nanjing 211816, P.R. China.

${ }^{\mathrm{b}}$ School of Environmental and Chemical Engineering, Jiangsu University of Science and Technology, Zhenjiang 212003, Jiangsu, P.R. China. 

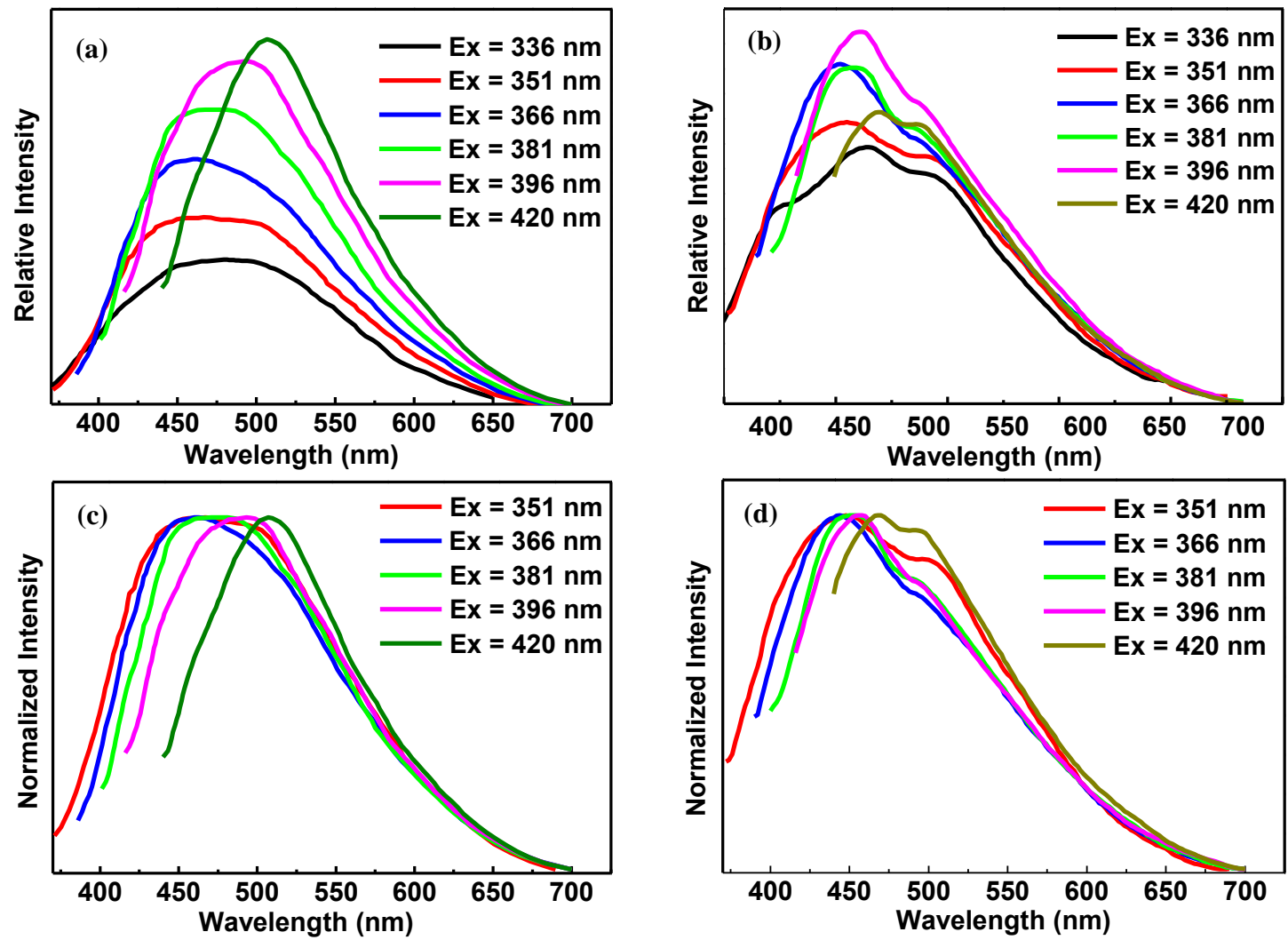

Figure S1. Solid-state emission spectra of Bmib ( $\mathrm{a}=$ measured, $\mathrm{c}=$ normalized) and IAM16-1 $(\mathrm{b}=$ measured, $d=$ normalized) and at various excitation wavelengths from 336 to $420 \mathrm{~nm}$ at $77 \mathrm{~K}$ (for normalized solid-state emission spectra of Bmib and IAM16-1, the bands excited by $336 \mathrm{~nm}$ have been removed to make it clearly show the red-shifted trend). 

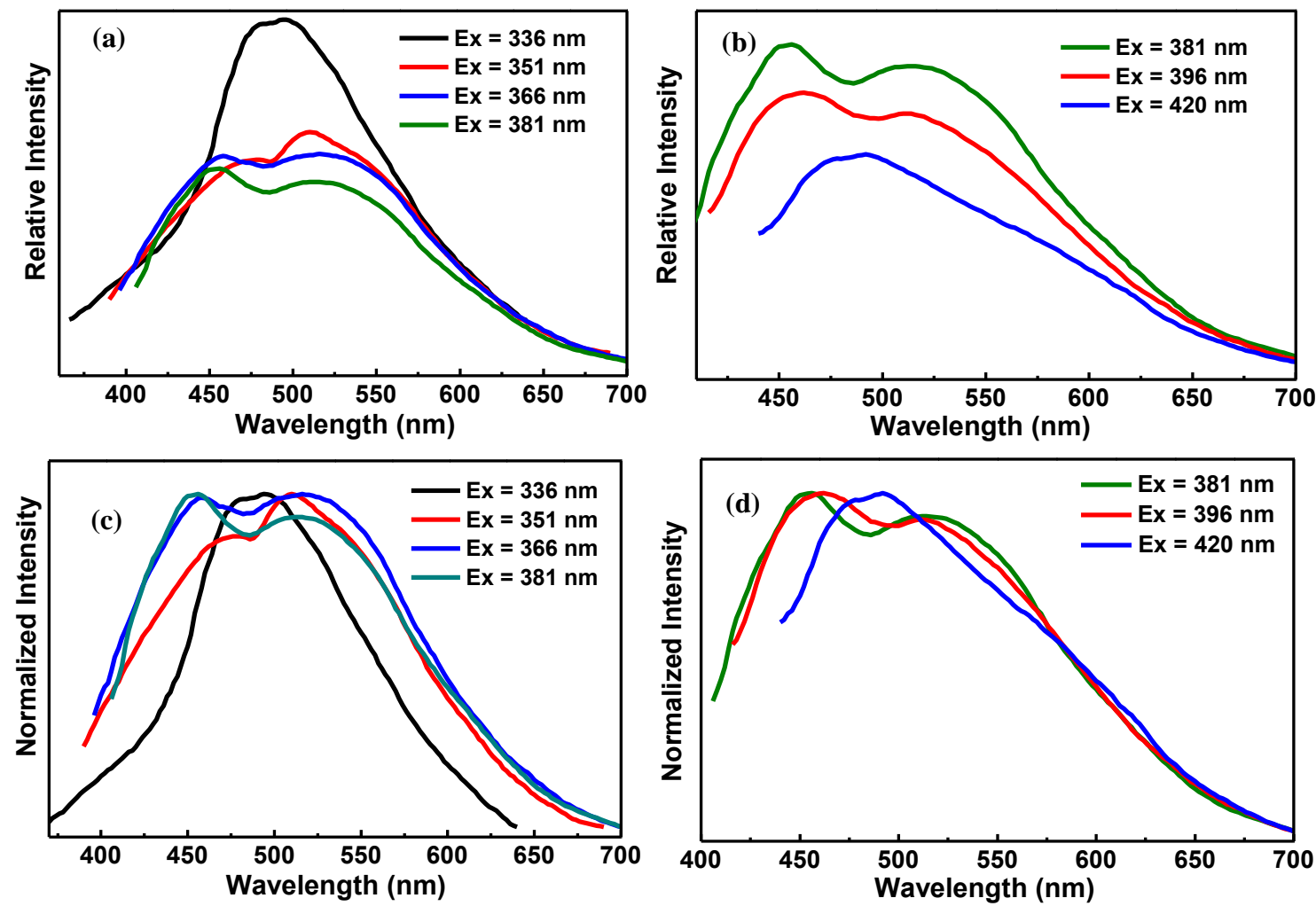

Figure S2. Solid-state emission spectra of IAM16-2 at various excitation wavelengths from 336 to $381 \mathrm{~nm}(\mathrm{a}=$ measured, $\mathrm{c}=$ normalized $)$, and from 381 to $420 \mathrm{~nm}(\mathrm{~b}=$ measured, $\mathrm{d}=$ normalized $)$ at $77 \mathrm{~K}$. 

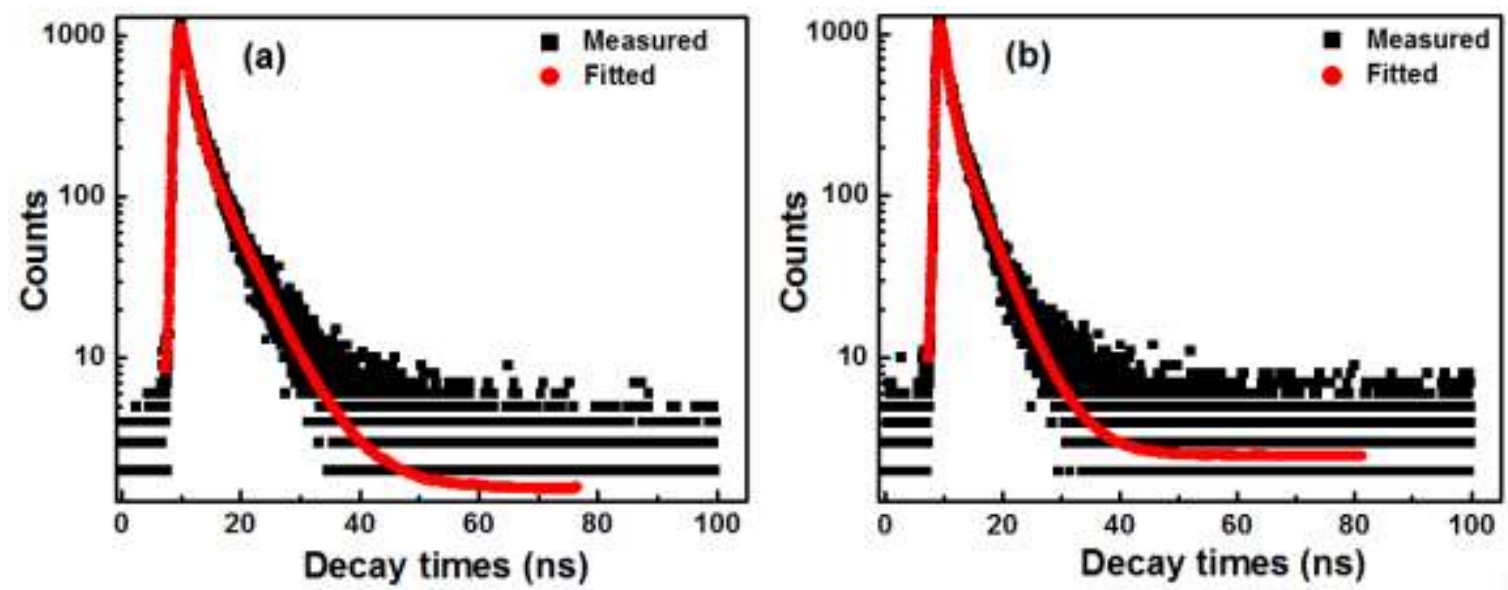

Figure S3. Fluorescence decay curves of IAM16-1 (a) and IAM16-2 (b) measured at room temperature.
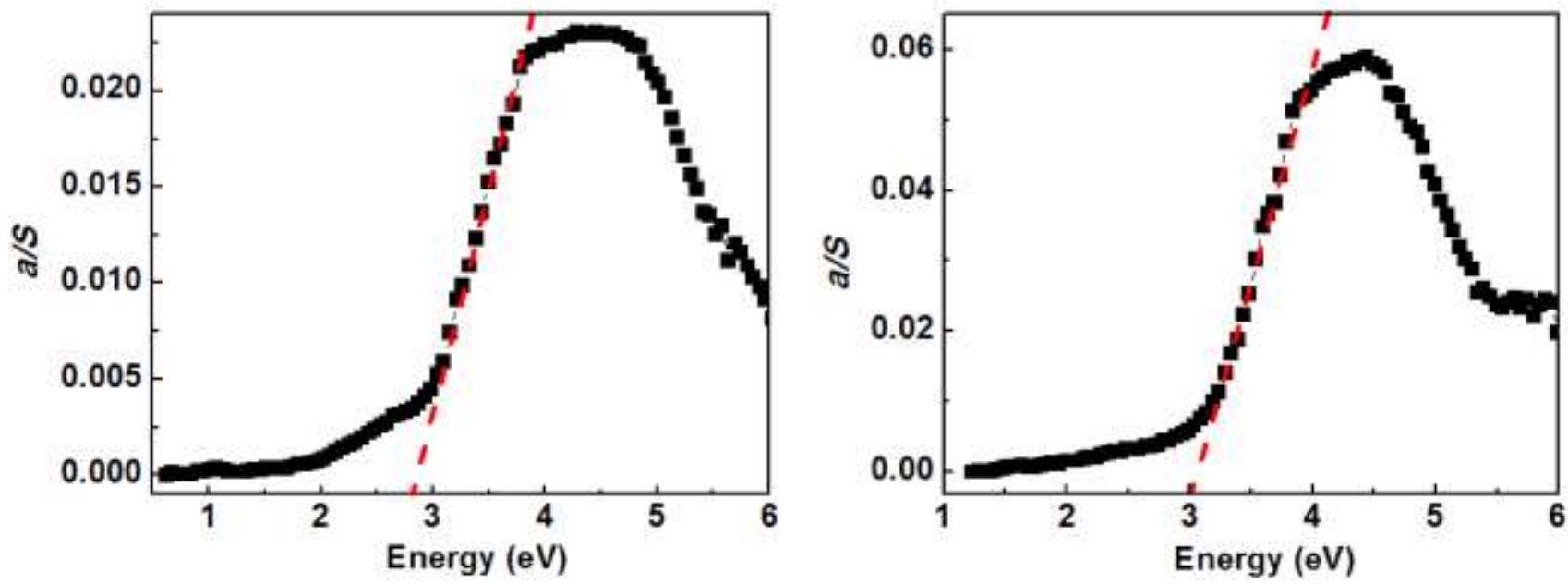

Figure S4. The experimental band gaps of IAM16-1 (left) and IAM16-2 (right) obtained from optical absorption spectra of them at room temperature. 


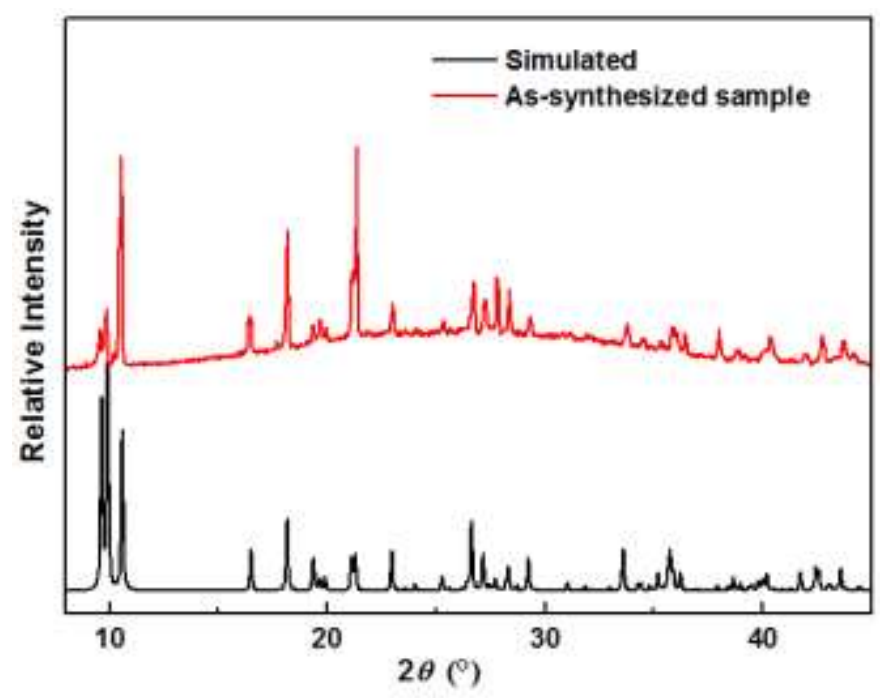

Figure S5. XRPD pattern simulated from a single crystal of IAM16-1 (black) and XRPD pattern of as-synthesized crystals of $\mathbf{I A M 1 6 - 1}$ (red).

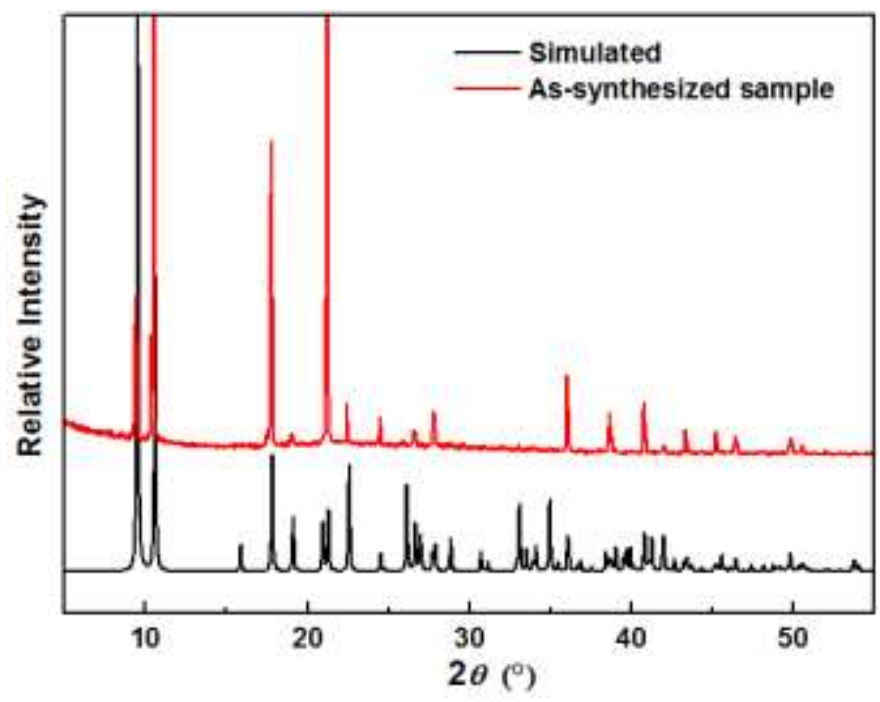

Figure S6. XRPD pattern of simulated from a single crystal of IAM16-2 (black) and XRPD pattern of as-synthesized crystals of $\boldsymbol{I A M 1 6 - 2}$ (red). 


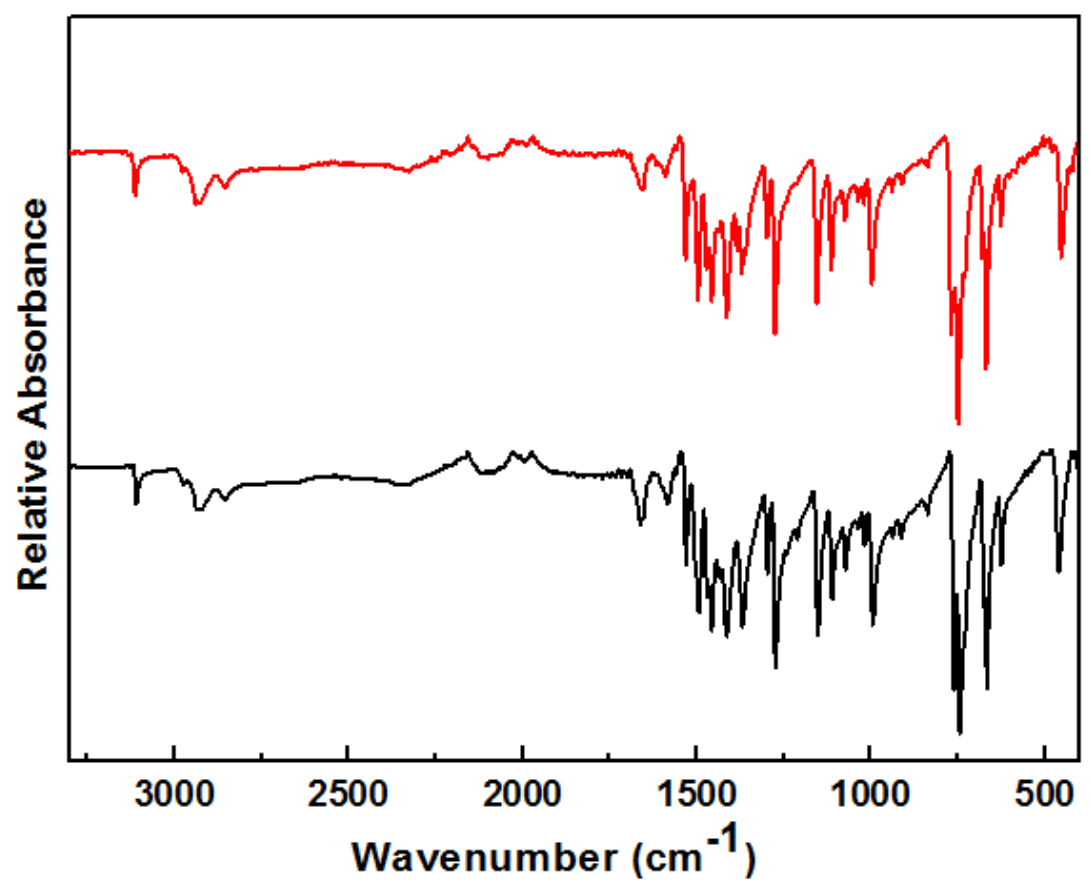

Figure S7. FT-IR spectra of IAM16-1 (black) and IAM16-2 (red).

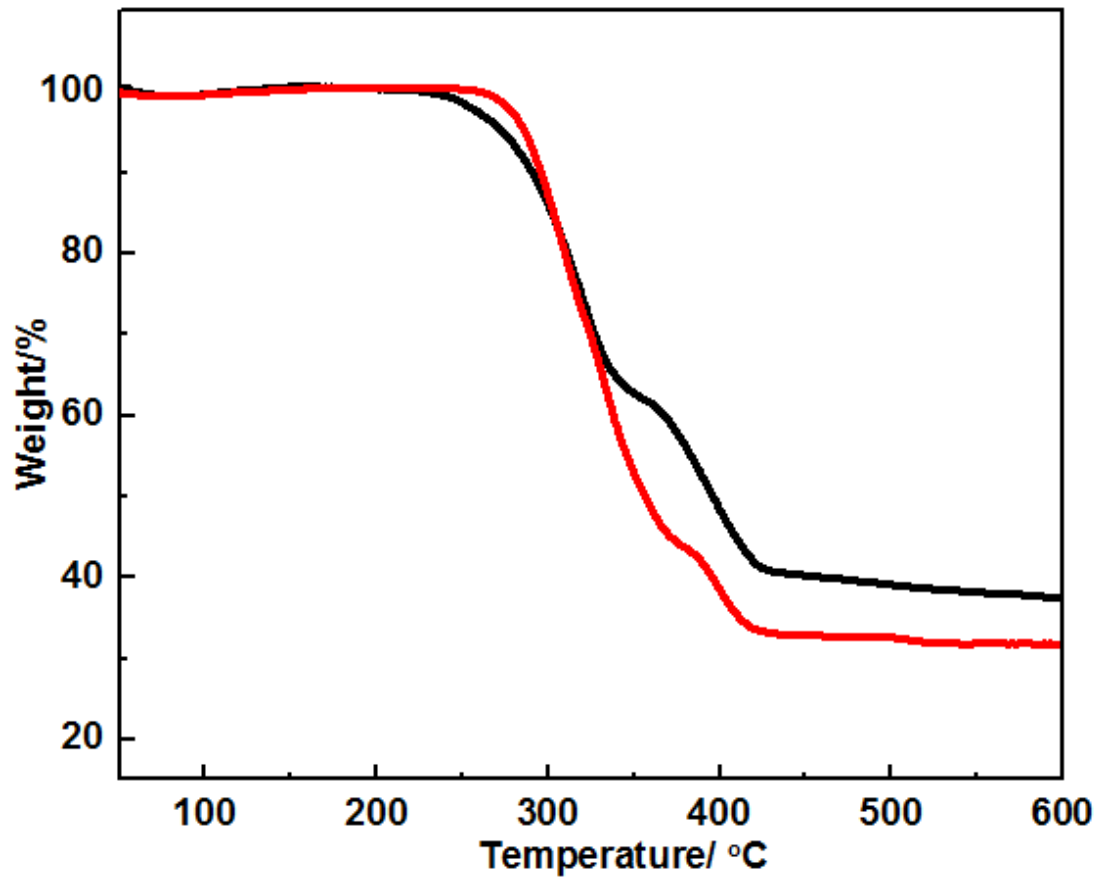

Figure S8. TGA profiles for IAM16-1 (black) and IAM16-2 (red). 
Table S1. The peak values of solid-state emission spectra of Bmib, IAM16-1 and IAM16-2 excited by the wavelengths ranging from $336 \mathrm{~nm}$ to $420 \mathrm{~nm}$ at $77 \mathrm{~K}$ (* denotes as the strongest peak of the band).

\begin{tabular}{|c|c|c|c|c|c|c|c|}
\hline \multirow{2}{*}{\multicolumn{2}{|c|}{\begin{tabular}{|c|} 
Sample Names \\
Measuring \\
Temperature
\end{tabular}}} & \multicolumn{2}{|c|}{ Bmib } & \multicolumn{2}{|c|}{ IAM16-1 } & \multicolumn{2}{|c|}{$I A M 16-2$} \\
\hline & & \multirow{2}{*}{\begin{tabular}{|c|}
$298 \mathrm{~K}$ \\
412
\end{tabular}} & \multirow{2}{*}{\begin{tabular}{|l|}
$77 \mathrm{~K}$ \\
448 \\
\end{tabular}} & \multirow{2}{*}{$\begin{array}{c}298 \mathrm{~K} \\
450\end{array}$} & \multirow{2}{*}{$\begin{array}{c}77 \mathrm{~K} \\
\begin{array}{c}404,460(*) \\
505\end{array}\end{array}$} & \multirow{2}{*}{$\begin{array}{c}298 \mathrm{~K} \\
493\end{array}$} & \multirow{2}{*}{$\begin{array}{l}77 \mathrm{~K} \\
489\end{array}$} \\
\hline \multirow{2}{*}{$\begin{array}{c}E x= \\
336(n m)\end{array}$} & $\operatorname{Em}(\mathrm{nm})$ & & & & & & \\
\hline & $\begin{array}{c}\text { Energy } \\
(\mathrm{eV})\end{array}$ & 3.02 & 2.77 & 2.76 & $3.08,2.70,2.46$ & 2.52 & 2.54 \\
\hline \multirow{2}{*}{$\begin{array}{c}E x= \\
351(\mathrm{~nm})\end{array}$} & $\operatorname{Em}(\mathrm{nm})$ & 436 & 451 & 434 & $449(*), 501$ & $463,507(*)$ & $477,501(*)$ \\
\hline & $\begin{array}{c}\text { Energy } \\
(\mathrm{eV})\end{array}$ & 2.85 & 2.75 & 2.86 & $2.77,2.48$ & $2.68,2.45$ & $2.60,2.48$ \\
\hline \multirow{2}{*}{$\begin{array}{c}\mathrm{Ex}= \\
366(\mathrm{~nm})\end{array}$} & $\operatorname{Em}(\mathrm{nm})$ & 447 & 461 & 440 & $444(*), 494$ & $446(*), 518$ & $458(*), 510$ \\
\hline & $\begin{array}{l}\text { Energy } \\
(\mathrm{eV})\end{array}$ & 2.78 & 2.70 & 2.82 & $2.80,2.51$ & $2.79,2.40$ & $2.71,2.44$ \\
\hline \multirow{2}{*}{$\begin{array}{c}\mathrm{Ex}= \\
381(\mathrm{~nm})\end{array}$} & $\operatorname{Em}(\mathrm{nm})$ & 454 & 470 & 450 & $453(*), 493$ & $449(*), 518$ & $453(*), 510$ \\
\hline & $\begin{array}{l}\text { Energy } \\
(\mathrm{eV})\end{array}$ & 2.74 & 2.64 & 2.76 & $2.74,2.52$ & $2.77,2.40$ & $2.74,2.44$ \\
\hline \multirow{2}{*}{$\begin{array}{c}E x= \\
396(\mathrm{~nm})\end{array}$} & $\mathrm{Em}(\mathrm{nm})$ & 469 & 493 & 462 & $458(*), 493$ & $461(*), 514$ & $462(*), 511$ \\
\hline & $\begin{array}{c}\text { Energy } \\
(\mathrm{eV})\end{array}$ & 2.65 & 2.52 & 2.69 & $2.71,2.52$ & $2.70,2.42$ & $2.69,2.43$ \\
\hline \multirow{2}{*}{$\begin{array}{c}E x= \\
420(n m)\end{array}$} & $\operatorname{Em}(\mathrm{nm})$ & 492 & 508 & 485 & $469(*), 493$ & 488 & 489 \\
\hline & $\begin{array}{c}\text { Energy } \\
(\mathrm{eV})\end{array}$ & 2.53 & 2.45 & 2.56 & $2.65,2.52$ & 2.55 & 2.54 \\
\hline \multicolumn{2}{|c|}{ Assignment } & \multicolumn{2}{|c|}{$\begin{array}{l}\text { ligand } \pi \\
\rightarrow \pi * \\
\text { transitions }\end{array}$} & $\begin{array}{c}\text { cluster-based } \\
\text { Br4p } \\
\rightarrow \text { Ag4d } \rightarrow \\
\text { ligand } \\
\text { (XMLCT) } \\
\text { charge transfer }\end{array}$ & $\begin{array}{c}\text { cluster-based } \\
\text { Br4p } \rightarrow \\
\text { Ag4d } \rightarrow \text { ligand } \\
\text { (XMLCT) } \\
\text { charge transfer }\end{array}$ & $\begin{array}{c}\text { cluster-based } \\
\text { I5p } \rightarrow \text { Ag4d } \rightarrow \\
\text { ligand }(\mathrm{XMLCT}) \\
\text { charge transfer }\end{array}$ & $\begin{array}{c}\text { cluster-based } \\
\text { I5p } \rightarrow \text { Ag4d } \rightarrow \\
\text { ligand } \\
\text { (XMLCT) } \\
\text { charge transfer }\end{array}$ \\
\hline
\end{tabular}

